\title{
Uplink Capacity of a Variable Density Cellular System with Multicell Processing
}

\author{
Efstathios Katranaras, Student Member, IEEE, Muhammad A. Imran, Member, IEEE, \\ and Costas Tzaras, Member, IEEE
}

\begin{abstract}
In this work we investigate the information theoretic capacity of the uplink of a cellular system. Assuming centralised processing for all Base Stations, we consider a power-law path loss model along with variable cell size (variable density of Base Stations) and we formulate an average path-loss approximation. Considering a realistic Rician flat fading environment, the analytical result for the per-cell capacity is derived for a large number of users distributed over each cell. We extend this general approach to model the uplink of sectorized cellular system. To this end, we assume that the user terminals are served by perfectly directional receiver antennas, dividing the cell coverage area into perfectly non-interfering sectors. We show how the capacity is increased (due to degrees of freedom gain) in comparison to the single receiving antenna system and we investigate the asymptotic behaviour when the number of sectors grows large. We further extend the analysis to find the capacity when the multiple antennas used for each Base Station are omnidirectional and uncorrelated (power gain on top of degrees of freedom gain). We validate the numerical solutions with Monte Carlo simulations for random fading realizations and we interpret the results for the real-world systems.
\end{abstract}

Index Terms-Information theory, Land mobile radio cellular systems, Multiaccess communication, Gaussian channels, Multipath channels, Propagation.

\section{INTRODUCTION}

The scientific field of information theory provides a mathematical framework which aims to quantify the maximum achievable data rate over a communication channel. Shannon [1] was the first to develop the underlying mathematical concepts that helped him predict the capacity of a single communication link and it took half a century of engineering to implement techniques which achieve capacities close to this prediction. Since then, communication systems have evolved from a simple transmitter-receiver link to complex ubiquitous communication systems driven by the desire of the users to be able to communicate while they move. The content that

E. Katranaras is with the Centre for Communications Systems Research, University of Surrey, Guildford, Surrey, GU2 7XH UK, e-mail: E.Katranaras@surrey.ac.uk.

M. A. Imran is with the Centre for Communications Systems Research, University of Surrey.

C. Tzaras is working for Kimatica Ltd, Greece.

Parts of this work were presented at IEEE SPAWC08 and IEEE PIMRC08 Efficiency Workshop. The work reported in this paper has formed part of the "Fundamental Limits to Wireless Network Capacity" Elective Research Programme of the Virtual Centre of Excellence in Mobile \& Personal Communications, Mobile VCE, www.mobilevce.com. This research has been funded by the following Industrial Companies who are Members of Mobile VCE - BBC, BT, Huawei, Nokia, Nokia Siemens Networks, Nortel, Vodafone. Fully detailed technical reports on this research are available to staff from these Industrial Members of Mobile VCE. needs to be communicated is not limited to voice only and the demand for high data rate services is growing fast. This revives the original question that Shannon posed for the single link, as a valid unanswered question for the communication systems of the new era.

In the last few decades, numerous attempts have been made to study the performance of cellular systems. Wyner [2] provided the insight for the performance of the cellular systems when the base-station receivers cooperate to jointly decode the received signals. This led to further research on the proposed joint decoding system. In [3], Wyner's simple yet tractable model (with a very crude approximation of path loss for the neighbouring cells users which were also assumed to be collocated with the base-stations) was extended to consider flat fading environments. The assumptions of fixed cell density (number of Base Stations in a unit area) and interfering adjacent cells were tackled only recently by Letzepis [4]. In this study, the one-dimensional (linear) part of Wyner's model was extended to incorporate distance dependent path loss and multiple-tier interference. The concept of sectorization in cellular systems has also been tackled in some previous studies ([5], [6]) without though considering joint processing at the receiver end.

In this work the linear system considered in [4] is extended to the two-dimensional (planar) one. The Shannon-theoretic limits of uplink cellular systems are presented by incorporating variable cell-sizes and fading in basic Wyner's model and the effect of using directional receiving antennas is analysed. In the presence of a power-law path loss and a general Rician flat fading environment, the effect of user spatial distribution on the capacity is investigated. More specifically, it is shown that the capacity is a function of the received signal power. Considering that the magnitude of the received signal power for each transmitted signal depends on the path loss between the transmitter and the receiver and that the specific user spatial distribution determines the values of the path loss coefficients for links between transmitters and receivers, one can say that for the same per transmitter power constraint, the magnitude of the power received at the Base Station (BS) of interest from all the cells (and consequently the capacity) depends on the user spatial distribution. Furthermore, multipletier interference is considered, providing insight in the role of the cell density on the capacity of a hyper-receiver considering Cellular Multiple Access Channel (MAC) system. We also show that the capacity formula for the cellular MAC with joint processing of all receivers, boils down to a Shannon-like formula. The parameter that controls the capacity given by this 
formula, is the ratio of the total amount of received power to the AWGN power at each receiver. This ratio is termed as the Rise over Thermal (RoT) and is shown to depend on several system parameters which are identified and analysed.

\section{A. Outline}

In Section II the system model is described and the basic assumptions are outlined. We present the path loss and the fading model used to represent the environment for our information-theoretic analysis. Furthermore, the generalised, the sectorized and the MIMO cellular system models are discussed. In Section III we present the average path loss approximation approach. The approach is analysed based on a conventional cellular system geometry and three different types of user spatial distribution over the cells are investigated with respect to their effect on the users' path loss coefficients. Section IV focuses on the capacity of the two cellular models and a tight upper bound is provided for the per-cell capacity in a realistic Rician fading environment, assuming large number of served users in every cell. The asymptotic capacity of the system is also found as the number of receiving antennas grows very large. Finally, Section V presents the theoretical and the practical (for the real-world systems) results and Section VI concludes the paper.

\section{B. Notation}

In the upcoming analysis, $N$ is the number of BSs in the cellular system, $K$ is the number of User Terminals (UTs) per cell and $\eta$ is the power-law path loss exponent. The index of the interference tier - a group of cells surrounding a specific cell at roughly the same distance from the specific cell - is $l$, while $L$ refers to the maximum number of tiers of interference considered for every cell. Each of the $N$ cells is labelled with a unique two-dimensional index vector: for cell $n$ this is defined as $\mathbf{v}_{n} \triangleq(p, q)$ where $p$ and $q$ are ordered row and column numbers, respectively, on the equivalent square grid obtained by the rotation and scaling of the hexagonal grid [2]. Throughout this paper, $\overline{(\cdot)}, \mathbb{E}[\cdot],(\cdot)^{*},(\cdot)^{T},(\cdot)^{\dagger}$, $\operatorname{det}[\cdot]$, diag $[\cdot]$ and $\odot$ denote the mean value, the expectation, the complex conjugate, the transpose matrix, the conjugate transpose matrix, the determinant of a matrix, the diagonal matrix and the Hadamard product respectively. The notation $a \gg b$ means that $a$ is much greater than $b$. The logarithmic expression $\log (x)$, unless stated otherwise, refers to the natural logarithm $\log _{e}(x)$. For the capacity calculations though, the binary logarithm $\log _{2}(x)=\frac{\log (x)}{\log (2)}$ is used.

\section{SySTEM MOdEL}

We assume a two-dimensional hexagonal cellular array and a network of cells where the BSs are uniformly distributed in a hexagonal grid. All the antennas are considered to be omnidirectional for the non-sectorized case while for the sectorized case the BS antennas are considered to be perfectly directional. A BS located at the center of each cell, will receive signals from all the users in the system, attenuated according to the power-law path loss and the multipath fading. We assume

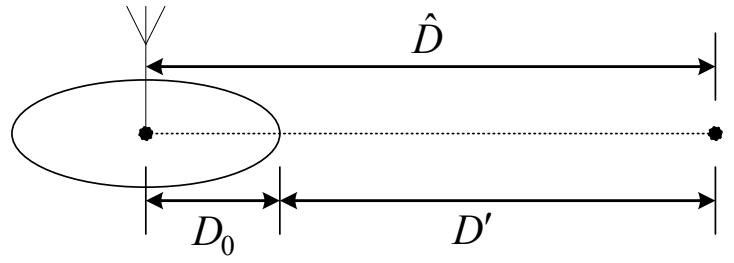

Fig. 1. Definitions of distances for path loss model

that all the BSs cooperate to jointly decode the received signals ("hyper-receiver" scheme). All the users of the system are sharing the same frequency and time resource and they are spatially distributed over the cells. Similar to Hanly's circular array model (for a linear system) [7] a wrap-around toric model is adopted (for a planar 2D system). In such a model, every cell has the same number of surrounding cells in order to avoid the edge effects. Nevertheless, for large number of cells the edge effects do not significantly affect the results [2].

\section{A. Path Loss and Fading Model}

1) Path Loss Model: A widely used model that maps the path gain (defined as the ratio of the received over the transmitted power) and the distance in a power-law path loss environment is expressed as:

$$
\varsigma^{2}=L_{0}\left(\frac{D_{0}}{\hat{D}}\right)^{\eta}
$$

where $\varsigma$ is the variance profile function describing the path loss environment, $L_{0}$ is defined as the power received at a reference distance $D_{0}$ when transmitted power is unity and $\eta$ is the power-law path loss exponent. The distance of the user terminal from the antenna is $\hat{D}$. Consider Fig. 1 where a reference point is located at a distance $D_{0}$ from the antenna. We can define the distance from the reference point to the user terminal as $D^{\prime}$. It is clear that $\hat{D}=D^{\prime}+D_{0}$. Making this substitution in (1), we get

$$
\varsigma^{2}=L_{0}\left(\frac{D_{0}}{D_{0}+D^{\prime}}\right)^{\eta}
$$

which can be rearranged to get

$$
\varsigma^{2}=\frac{L_{0}}{\left(1+\frac{D^{\prime}}{D_{0}}\right)^{\eta}}=\frac{L_{0}}{(1+D)^{\eta}}
$$

with $D=\frac{D^{\prime}}{D_{0}}$ defined as the normalised distance. We can assume $L_{0}$ to be scaled to unity for simplifying the analytical approach. If the distance between a user $k$ in a cell $m$ from the reference point in cell $n$ is defined as $D_{m, k}^{(n)}$, the power-law path gain from the user $k$ to the receiver of cell $n$ is expressed as:

$$
\varsigma_{m, k}^{(n)}=\left(1+D_{m, k}^{(n)}\right)^{-\eta / 2}
$$

The modified path loss model of (4) is not a perfect representation for practical systems, but its a close approximation and can serve as a useful tool for information-theoretic analysis. 
2) Fading model: Considering the uniformly distributed random received phase $\Phi$ on the specular path, a generalised model for the fading coefficients can be given by [8], [9], [10]:

$$
g=\sqrt{\frac{\kappa}{\kappa+1}} \mathrm{e}^{j \Phi}+\sqrt{\frac{1}{\kappa+1}} \mathcal{C N}(0,1)
$$

where $\mathbb{E}[g g *]=1, \kappa$ is used to define the ratio of the power in the specular path and the non-specular multipaths and $\mathcal{C N}(0,1)$ represents a complex Gaussian random variable with independent real and imaginary components each normally distributed with mean zero and variance $1 / 2$.

It shall be pointed out that the mean value of the product of two independent fading realisations is zero if the received signals on the specular path have uniformly distributed random phase offsets. Furthermore, the Rayleigh fading environment (no specular component) is, by its nature, a zero mean environment and the expected value of each fading coefficient in this case is zero.

\section{B. Generalised Cellular System Model}

Consider a network of $N$ cells and $K$ users in each cell. According to our model, the received signal at the BS antenna of cell $n$ is the sum of the transmitted signals from the users within the same cell and also from the rest of the cells in the system (appropriately scaled by the path gain and fading coefficients). Hence, the received signal in a cell $n$ is given by:

$$
\begin{aligned}
y_{n}=\sum_{k=1}^{K}\left[\varsigma_{n, k}^{(n)}\right. & \left.\cdot g_{n, k}^{(n)} \cdot x_{n, k}\right]+ \\
& +\sum_{\substack{m=1 \\
m \neq n}}^{N} \sum_{k=1}^{K}\left[\varsigma_{m, k}^{(n)} \cdot g_{m, k}^{(n)} \cdot x_{m, k}\right]+z_{n}
\end{aligned}
$$

Where $y_{n}$ and $z_{n}$ represent the received signal and the AWGN noise (normalised to unit power) at the receiver of cell $n$. The variable $x_{m, k}$ represents the complex Gaussian inputs for a transmitter $k$ in cell $m$ and $\varsigma_{m, k}^{(n)}, g_{m, k}^{(n)}$ represent the path gain coefficients and the fading coefficients between a transmitter $k$ in cell $m$ and the receiver at the BS of cell $n$. All the complex fading coefficients are normalized to unit power and when viewed as complex random processes are circularly symmetric i.i.d. Gaussian, stationary and ergodic. It is assumed that each user has average power constraint $P$, i.e. $\mathbb{E}\left[x_{m, k} \cdot x_{m, k}^{*}\right] \leq$ $P$.

\section{Sectorized Cellular System Model}

Consider now a two dimensional network of $N, 3$-sectored cells, with $\ddot{K}=K / 3$ users uniformly divided into each sector. Label the sectors as $(A),(B)$ and $(C)$ as shown in Fig. 2. The receiver at sector $(A)$ of a cell $n$ will receive signals from the shaded area illustrated in the same figure. We assume perfect directional antennas at the BSs which means that each antenna exclusively covers (receives signals from) one third

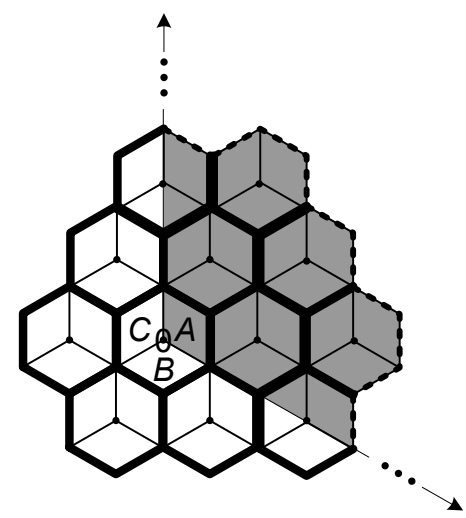

Fig. 2. The sectorized cellular system model. The shaded area denotes the area of interference for the sector- $(A)$-receiver at the cell of interest.

of the system users. Hence, sector- $(A)$-received signal, at cell $n$, is given by:

$$
\begin{aligned}
& y_{n}^{(A)}=\sum_{k \in \mathcal{K}_{n,(A)}^{n}}\left[\sqrt{G_{D,(3)} \varsigma_{n, k}^{(n)}} g_{n, k}^{(n),(A)} x_{n, k}\right] \\
& +\sum_{m \in \mathcal{N}_{(A)}^{n}} \sum_{k \in \mathcal{K}_{m,(A)}^{n}}\left[\sqrt{G_{D,(3)}} \varsigma_{m, k}^{(n)} g_{m, k}^{(n),(A)} x_{m, k}\right]+z_{n}^{(A)}
\end{aligned}
$$

where $G_{D,(S)}$ (with $1 \leqslant G_{D,(3)} \leqslant 3$ ) is the directivity power gain of each of the $S$ receiving antennas used at the BSs and the rest variables are defined as for the non-sectored case. The additional superscript also identifies the specific sector-antenna at the receiver end. Set $\mathcal{K}_{m,(A)}^{n}$ describes the subset of users in any cell $m$ that are in the coverage area of the sector $(A)$ of cell $n$ (shaded area in Fig. 2). Set $\mathcal{N}_{(A)}^{n}$ is the subset of all cells (excluding cell $n$ ) that are in the coverage area of the sector $(A)$ of the cell $n$.

We have to note here that we have assumed directional antennas receiving only in the horizontal plane, without taking into consideration of the vertical plane, which is the fairest for comparing with the omnidirectional antenna case. Nevertheless, this assumption does not lead to a loss of generality since the antenna gain is defined straightforwardly as the antenna directivity times the factor representing the antenna efficiency and can be easily accessed in the following analysis according to any antenna scenario given. In our case, a $100 \%$ efficient directional antenna for a 3-sectored system will have a gain equal to its directivity, which is 3 when taking into consideration only the horizontal plane. In the contrary to the previous best case scenario, when the directional antenna is not efficient at all, the gain will be 1. For more details on directive antennas one can refer to [6] and references therein.

\section{MIMO Cellular System Model}

Consider the same network as above except that in every BS now we have three omnidirectional receiving antennas which are not correlated with each other. In that case, the received signal at $i^{t h} \mathrm{BS}$ antenna in a cell $n$ is given by the modified 


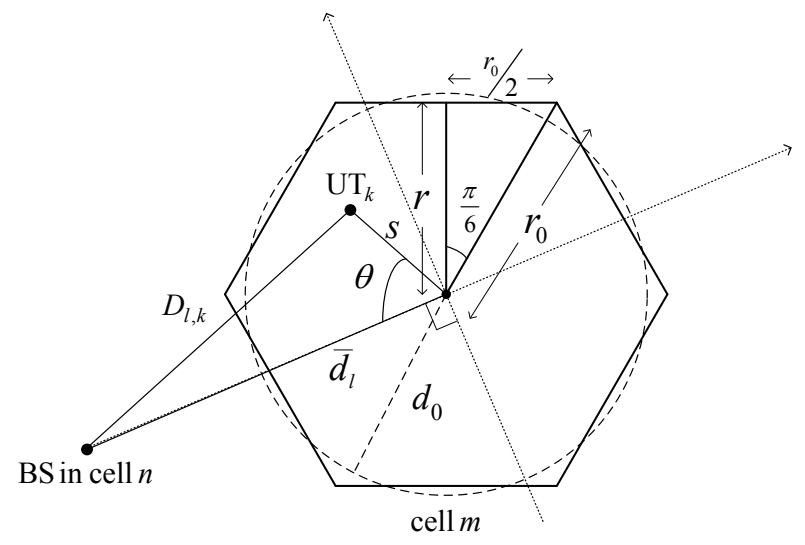

Fig. 3. Geometry of a regular hexagonal cell with side length of $r_{0}$, the circular equivalent of radius $d_{0}$ and the distance $D_{l, k}$ of a User Terminal in a cell $m$ from a Base Station in a cell $n$. Cell $m$ is considered to be at the $l^{\text {th }}$ tier of interference w.r.t. cell $n$.

form of (6):

$$
\begin{aligned}
y_{n}^{i}=\sum_{k=1}^{K}\left[\varsigma_{n, k}^{(n)} \cdot g_{n, k}^{(n),(i)} \cdot x_{n, k}\right]+ \\
\quad+\sum_{\substack{m=1 \\
m \neq n}}^{N} \sum_{k=1}^{K}\left[\varsigma_{m, k}^{(n)} \cdot g_{m, k}^{(n),(i)} \cdot x_{m, k}\right]+z_{n}^{(i)}
\end{aligned}
$$

where $g_{m, k}^{(n),(i)}$ represents the fading coefficient between the user $k$ in cell $m$ and the receive antenna $i$ at the BS of cell $n$.

\section{Average Path Loss Approximation Approach}

\section{A. Cell and System Geometry}

Consider a regular hexagonal cell with its geometry given in Fig. 3. The side of the regular hexagon is denoted by $r_{0}$ and the minimal radius of the hexagon is $r=r_{0} \cdot \cos \left(\frac{\pi}{6}\right)$. Here, we can define the Inter Site Distance (ISD) as the distance between two adjacent BSs (ISD $\triangleq 2 r$ ), which will be extensively used later on the paper. In our planar cellular system model, we assume multiple tiers of interference around each cell as shown in Fig. 4. The irregular boundary of each tier can be represented by an equivalent regular hexagon with the length of its side given by:

$$
r_{l}=\sqrt{[(2 l+1) \cdot r]^{2}+\left(\frac{r_{0}}{2}\right)^{2}}
$$

where $l$ stands for the $l^{t h}$ tier of interference.

In general, the hexagonal (with side length $r_{l}$ ) boundary of any tier can be approximated by an equivalent circular boundary, as shown in Fig. 3. The equivalence is in the sense that the average distance of all points on the perimeter of the two shapes (circle and the hexagon) is same. The radius of such an equivalent circular boundary of any tier, is given by:

$$
d_{l}=\frac{6}{\pi} \int_{0}^{\frac{\pi}{6}} \frac{r_{l}}{\cos \theta} \cos \left(\frac{\pi}{6}\right) d \theta
$$

For evaluating the capacity under any user spatial distribution with large number of users, it is useful to group the users in

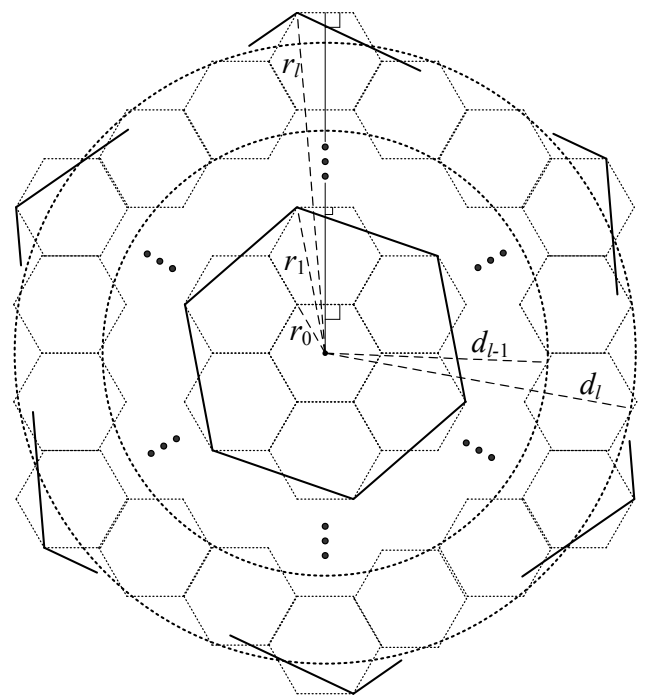

Fig. 4. Multiple tiers of interference around a cell. The irregular boundary of each tier can be represented by an equivalent regular hexagon and the latter by a circular boundary.

each interference tier and represent their squared path gain coefficients with an appropriate mean value, denoted by $\overline{\varsigma_{l}^{2}}$. This mean value is calculated by focusing on a single cell and averaging the path loss of all users in this cell, with reference to the receiver position. This average can be expressed as a function of the distance between the center of the cell in focus and the receiver. As the distance of various cells in a given interference tier slightly vary from one cell to the other, we can further estimate this distance using an approximate distance $\bar{d}_{l}$, from the inner and outer circular boundary of the $l^{\text {th }}$ tier of interference (see Fig. 4):

$$
\bar{d}_{l} \approx \frac{d_{l}+d_{l-1}}{2} .
$$

It is worth to note here that an alternative wedge-cell geometry is proposed in [11] and [6]. Although there are similarities on the two approaches the wedge-cell geometry may be less efficient in approximating the uniform spatial distribution of users as it assumes more users closer the edge of the cells.

\section{B. User Distribution and Mean Squared Path Loss}

The mean squared path loss for the user terminals in a cell will depend on the proximity (which interference tier the cell belongs to) of the cell to the receiver of interest and also on the user spatial distribution over the cell. We define the distance $D_{l, k}$ of a user in a cell in the $l^{\text {th }}$ tier of interference from the receiver of interest. With the help of Fig. 3, one can prove that:

$$
D_{l, k}(\theta, s)=\sqrt{\left(\bar{d}_{l}-s \cdot \sin \theta\right)^{2}+(s \cdot \cos \theta)^{2}}
$$

where $s$ and $\theta$ respectively define the radial and angular location of a User Terminal (UT), with respect to the receiver of a BS as shown in Fig. 3. Three different cases of user spatial distribution are examined in this paper.

Uniform Distribution: In this case, the users are assumed to be uniformly distributed over the planar system. The mean 
squared path gain for each of the $K$ users in a cell which belongs in the $l^{\text {th }}$ tier of interference from the receiver of interest is given by:

$$
\overline{\varsigma_{l-\text { uni }}^{2}}=\frac{1}{\pi \cdot d_{0}^{2}} \int_{0}^{d_{0}} \int_{-\pi}^{\pi} \frac{1}{\left(1+D_{l, k}(\theta, s)\right)^{\eta}} \cdot s \cdot d \theta d s
$$

Uniform spatial distribution represents a likely distribution in a real-world system when a large number of users is considered to be randomly placed over the system.

Truncated Cell-Centre Uniform Distribution: Here, the users are uniformly distributed around the centre of their cell and thus,

$$
\overline{\varsigma_{l-c e n t r e}^{2}}=\frac{1}{\pi \cdot \rho^{2}} \int_{0}^{\rho} \int_{-\pi}^{\pi} \frac{1}{\left(1+D_{l, k}(\theta, s)\right)^{\eta}} \cdot s \cdot d \theta d s
$$

where $\rho$ (with $0<\rho \leq d_{0}$ ) is the truncation radius around each BS in which the $K$ users are distributed. Note that for values of $\rho$ very close to zero (users are co-located with each $\mathrm{BS})$, the mean squared path gain approaches to $\frac{1}{\left(1+\bar{d}_{l}\right)^{\eta}}$.

Truncated Cell-Edge Uniform Distribution: In this last case, the users are uniformly distributed on an annular segment close to the edge of their cell. We have,

$$
\overline{\varsigma_{l-e d g e}^{2}}=\frac{1}{\pi \cdot\left(d_{0}^{2}-\dot{\rho}^{2}\right)} \int_{\dot{\rho}}^{d_{0}} \int_{-\pi}^{\pi} \frac{1}{\left(1+D_{l, k}(\theta, s)\right)^{\eta}} s d \theta d s
$$

where $\dot{\rho}$ (with $0 \leq \dot{\rho}<d_{0}$ ) is the radial distance from the center of the cell to the boundary where the annular section (on which the users are distributed) starts. Note that for values of $\rho$ very close to $d_{0}$ (all users are very close to the edge of the cell), the mean squared path gain can be assumed to be given by $\frac{1}{\pi} \int_{-\frac{\pi}{2}}^{\frac{\pi}{2}} \frac{1}{\left(1+D_{l, k}(\theta, s)\right)^{\eta}} d \theta$.

Note that the transmitted signals from the users in the cell of interest also follow the same power-law path loss described above. For this case $\bar{d}_{l}=0$ and it follows from (12) that $D_{l, k}(\theta, s)$ becomes $s$ for the path loss calculations using the above analysis.

\section{CAPACITY ANALYSIS}

\section{A. Generalised Cellular Model}

The output vector of all the received signals in the system can be given using the channel equation (6), as:

$$
\mathbf{y}=\mathbf{H x}+\mathbf{z}
$$

where $\mathbf{y}=\left[y_{1}, y_{2}, \ldots y_{N}\right]^{T}$ is the $N \times 1$ received signal column vector, $\mathbf{x}=\left[\mathbf{x}_{1}^{T}, \mathbf{x}_{2}{ }^{T}, \ldots \mathbf{x}_{N}{ }^{T}\right]^{T}$ is the $N K \times 1$ column vector of the transmitted signals of all the users, with $\mathbf{x}_{n}=\left[x_{n, 1}, \ldots, x_{n, K}\right]^{T}$, denoting the concatenation of the transmitted signals from the $K$ users in cell $n, \mathbf{z}$ is the $N \times 1$ column vector of noise and $\mathbf{H}$ is the overall $N \times N K$ system gain matrix given by:

$$
\mathbf{H}=\mathbf{\Sigma} \odot \mathbf{G}
$$

where $\boldsymbol{\Sigma}$ is a deterministic $N \times N K$ matrix that contains all the path gain coefficients of the channels and $\mathbf{G}$ is the $N \times N K$ matrix of all the fading coefficients. In $\mathbf{H}$ matrix, each row

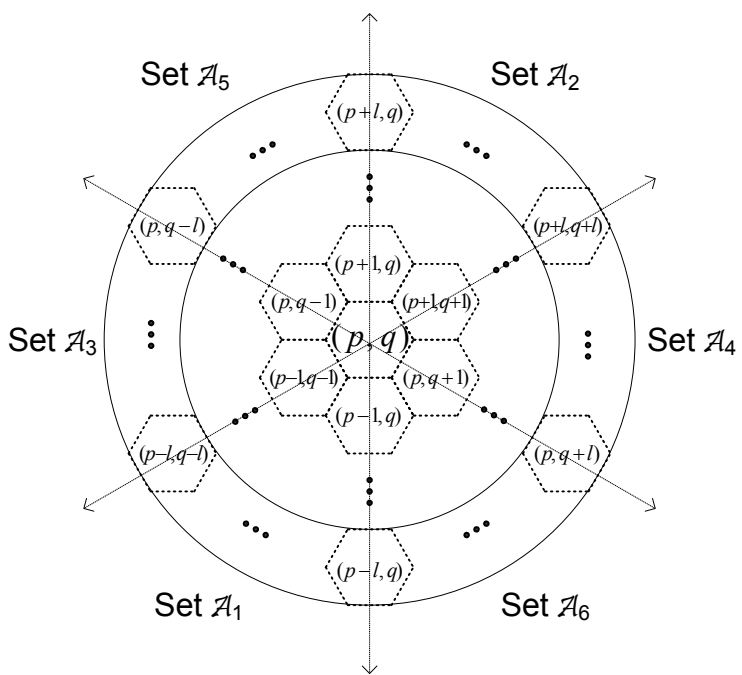

Fig. 5. The six sets describing which cells belong to the $l^{\text {th }}$ tier of interference around a cell $(p, q)$. Two indices describe the position of each cell in the planar system w.r.t. the cell of interest.

corresponds to a specific receiver and each column to a specific transmitter.

For describing the matrices formulated above, consider the representation of the cellular system as a rectangular array, as described by Wyner in [2], and the raster scanning method that was used by Somekh and Shamai in [3] to define the order of the system output vector elements (i.e. the one-to-one mapping of all two dimensional index vectors for the cells to a unique one dimensional index system). Considering a specific wrap-around toric model, $\boldsymbol{\Sigma}$ can be considered as a blockcirculant matrix, in terms of its row-vector elements. Assume the $n^{t h}$ row corresponding to the receiver of cell $n$ has the two dimensional index given as $\mathbf{v}_{n}=(p, q)$. There are six subsets the union of which describes the cells that belong to the $l^{\text {th }}$ tier of interference around the cell of interest. Subsequently, they describe the row blocks that contain the appropriate path gain coefficients (see Fig. 5):

$$
\begin{aligned}
& \mathcal{A}_{1} \triangleq\{(p-l, q-l),(p-l, q-l+1), \cdots,(p-l, q)\} \\
& \mathcal{A}_{2} \triangleq\{(p+l, q),(p+l, q+1), \cdots,(p+l, q+l)\} \\
& \mathcal{A}_{3} \triangleq\{(p-l, q-l),(p-l+1, q-l), \cdots,(p, q-l)\} \\
& \mathcal{A}_{4} \triangleq\{(p, q+l),(p+1, q+l), \cdots,(p+l, q+l)\} \\
& \mathcal{A}_{5} \triangleq\{(p, q-l),(p+1, q-l+1), \cdots,(p+l, q)\} \\
& \mathcal{A}_{6} \triangleq\{(p-l, q),(p-l+1, q+1), \cdots,(p, q+l)\}
\end{aligned}
$$

The maximum per-cell capacity is achieved when all UTs are allowed to transmit all the time at their maximum transmit power constraint (Wideband scheme presented in [3]), and this capacity is given by [12]:

$$
C=\lim _{N \rightarrow \infty} \mathbb{E}\left[\frac{1}{N} \log \operatorname{det}\left(\boldsymbol{\Lambda}_{\mathbf{y}}\right)\right]
$$

where the expectation is taken over all the fading realizations and $\Lambda_{\mathbf{y}}$ is the covariance matrix of the system output vector:

$$
\boldsymbol{\Lambda}_{\mathbf{y}}=P \cdot \mathbf{H H}^{\dagger}+\mathbf{I}_{N \times N}
$$


Considering that the number of cells grows very large, $\boldsymbol{\Lambda}_{\mathbf{y}}$ becomes a large random matrix. We use Jensen's inequality that provides an upper bound for the capacity of the system as:

$$
\lim _{N \rightarrow \infty}\left(\frac{1}{N} \log \left(\operatorname{det} \mathbb{E}\left[\boldsymbol{\Lambda}_{\mathbf{y}}\right]\right)\right) \geqslant \lim _{N \rightarrow \infty} \mathbb{E}\left[\frac{1}{N} \log \operatorname{det}\left(\boldsymbol{\Lambda}_{\mathbf{y}}\right)\right]
$$

Assuming that the number of UTs per cell is growing large for a fixed number of cells, $K N$ tends to infinity. In this case, the law of large numbers (that describes the long-term stability of the elements of the covariance matrix when the number of users per cell is large) ensures that the upper bound presented above is tight [3] (see Appendix A). Hence,

$$
C \leq \lim _{N \rightarrow \infty}\left(\frac{1}{N} \log \left(\operatorname{det} \mathbb{E}\left[\boldsymbol{\Lambda}_{\mathbf{y}}\right]\right)\right) \text { for } K \gg 1
$$

1) Capacity in Rician fading environment: As the fading coefficients are assumed normalized to unit power, we have:

$$
\mathbb{E}\left[g_{m, k}^{(n)} \cdot\left(g_{m, k}^{(n)}\right)^{*}\right]=\mathbb{E}\left[\left(g_{m, k}^{(n)}\right)^{2}\right]=1
$$

Furthermore, when a Rician fading environment is assumed, the received signals on the specular path have uniformly distributed random phase offsets. If we consider that the fading coefficients referring to different channels are independent, it follows from (5) that:

$$
\mathbb{E}\left[g_{m, k}^{(n)} \cdot\left(g_{\dot{m}, k}^{(\hat{n})}\right)^{*}\right]=0
$$

Under these assumptions, the expectation of the covariance matrix of the output vector converges to a diagonal matrix. If we assume uniform user spatial distribution, a maximum of $L$ tiers of interference for every cell and consider that there are always $6 \cdot l$ cells in the $l^{\text {th }}$ tier of interference, the $N \times N$ expectation matrix becomes :

$\mathbb{E}\left[\boldsymbol{\Lambda}_{\mathbf{y}-\text { uni }}\right]=\operatorname{diag}\left[1+K P \cdot\left(\overline{\varsigma_{0-u n i}^{2}}+\sum_{l=1}^{L} 6 \cdot l \cdot \overline{\varsigma_{l-u n i}^{2}}\right)\right]$

where $\overline{\varsigma_{0-\text { uni }}^{2}}$ denotes the mean squared path gain for the users inside the cell of interest. Taking into consideration equations (22) and (25), the asymptotic expression of the maximum percell capacity for any finite number of users $K$ is given by:

$$
\begin{aligned}
C_{\text {uni }} & =\lim _{N \rightarrow \infty} \frac{1}{N} \log \operatorname{det}\left(\mathbb{E}\left[\boldsymbol{\Lambda}_{\mathbf{y}-\text { uni }}\right]\right)= \\
& =\log \left[1+K P \cdot\left(\overline{\varsigma_{0-\text { uni }}^{2}}+\sum_{l=1}^{L} 6 \cdot l \cdot \overline{\varsigma_{l-\text { uni }}^{2}}\right)\right]
\end{aligned}
$$

Following the same procedure for the other two types of spatial distribution, one can easily reach to similar expressions for the per-cell capacity.

2) Capacity in Shadow fading environment: We consider the relevance of our model in a shadow fading environment. In the presence of shadow fading, the ratio of transmit to received power $(\psi)$, for a fixed distance between the transmitter and the receiver, can be modelled as a log-normal random variable with the following distribution [10]: $p(\psi)=\frac{\xi}{\sqrt{2 \pi} \sigma_{\psi d B} \psi} \exp \left[-\frac{\left(10 \log _{10} \psi-\mu_{\psi d B}\right)^{2}}{2 \sigma_{\psi d B}^{2}}\right], \psi>0$

where $\xi=\frac{10}{\ln 10}, \mu_{\psi d B}$ is the mean value of the variable $\psi_{d B}=$ $10 \log _{10} \psi$ and $\sigma_{\psi d B}$ is the standard deviation of the same variable. The linear average of random variable $\psi$ can be found from (27) as given below [10]:

$$
\mu_{\psi}=\mathbb{E}[\psi]=\exp \left[\frac{\mu_{\psi d B}}{\xi}+\frac{\sigma_{\psi d B}^{2}}{2 \xi^{2}}\right]
$$

When distance is also varying, $\mu_{\psi d B}$ becomes a function of distance,

$$
\mu_{\psi}(d)=\exp \left[\frac{\mu_{\psi d B}(d)}{\xi}+\frac{\sigma_{\psi d B}^{2}}{2 \xi^{2}}\right]
$$

where $\mu_{\psi d B}(d)$ accounts for the propagation loss due to the distance as well as the loss due to blockage caused by shadowing obstacles.

Considering the above model and a large number of independent users at each distance (so that the law of large numbers can be invoked), the mean received power can be modelled as a deterministic function of distance and shadow fading standard deviation. Considering shadow fading environment, $\mu_{\psi}$ (the linear mean value of transmit to receive power ratio) is by definition same as the calculated variable $\overline{\varsigma^{2}}$ in the absence of shadowing. Recall that we assume that the power gain for the Rayleigh/Rician fading component is normalised to unity. Since random variations in the ratio of transmit to receive power for any given distance are cancelled out when law of large numbers can be invoked, the mean value of the ratio becomes more important for the calculation.

An alternative approach to study the capacity of the shadow fading case is to capture the mean value of this ratio in the empirical propagation model given in (1) using the parameters $L_{0}$ and $\eta$. Since shadowing is essentially a loss in received power, smaller received power at the reference point and a larger path loss exponent can capture the essence of the shadow fading. These values can be determined using the curve-fitting approach for a given set of measurement data. Hence we can focus on the simplified path loss model for the analysis of capacity of the proposed system setup and this can still provide valid insights for the shadow fading scenario.

\section{B. Sectorized Cellular Model}

The system output vector in this case can be written based on (7) as:

$$
\mathbf{y}=\ddot{\mathbf{H}} \mathbf{x}+\mathbf{z}
$$

where $\mathbf{y}=\left[y_{1}^{(A)}, y_{1}^{(B)}, y_{1}^{(C)}, y_{2}^{(A)}, \ldots y_{N}^{(B)}, y_{N}^{(C)}\right]^{T}$ is the $3 N \times 1$ received signal column vector and the vector $\mathbf{x}=$ $\left[\mathbf{x}_{1}^{(A)}, \mathbf{x}_{1}^{(B)}, \mathbf{x}_{1}^{(C)}, \mathbf{x}_{2}^{(A)}, \ldots \mathbf{x}_{N}^{(B)}, \mathbf{x}_{N}^{(C)}\right]^{T}$ is the concatenation of the transmitted signals of all the users to form a $3 N \ddot{K} \times 1$ column vector, with $\mathbf{x}_{n}^{(i)}=\left[x_{n, 1}^{(i)}, \ldots, x_{n, \ddot{K}}^{(i)}\right]$ denoting the row concatenation of the transmitted signals of the $\ddot{K}$ users of 
sector $(i)$ in cell $n, \mathbf{z}$ is the $3 N \times 1$ noise column vector and, $\ddot{\mathbf{H}}$ is the $3 N \times 3 N \ddot{K}$ overall system gain matrix which is given by:

$$
\ddot{\mathbf{H}}=\sqrt{G_{D,(3)}} \cdot \ddot{\boldsymbol{\Sigma}} \odot \ddot{\mathbf{G}}
$$

where $\ddot{\Sigma}$ is a deterministic $3 N \times N K$ matrix that contains all the path gain coefficients of the system channels and $\ddot{\mathbf{G}}$ is the $3 N \times N K$ matrix of all fading coefficients. Based on the channel definition (30) and using again the raster scan method, the overall path gain matrix $\ddot{\Sigma}$ is a block-circulant matrix (in terms of its row-vector elements). In $\ddot{\Sigma}$ matrix, each row corresponds to a specific sector and the groups of three rows to a specific cell.

Following the discussion above for the generalised case, the maximum achievable capacity is given by:

$$
C \leq \lim _{N \rightarrow \infty}\left(\frac{1}{N} \log \left(\operatorname{det} \mathbb{E}\left[\ddot{\Lambda}_{\mathbf{y}}\right]\right)\right) \text { for } K \gg 1
$$

where the expectation is taken over all the fading realizations and $\ddot{\Lambda}_{\mathrm{y}}$ is the covariance matrix of the output vector of all the received signals in the system. This covariance matrix is given by:

$$
\ddot{\Lambda}_{\mathbf{y}}=P \cdot \ddot{\mathbf{H}} \ddot{\mathbf{H}}^{\dagger}+\mathbf{I}_{3 N \times 3 N}
$$

For the 3-sector cellular system model presented above, it can be observed that the expectation of the covariance matrix of the output vector is a block-circulant matrix with $3 \times 3$ blocks. We proceed to formulate the capacity of this sectorized cellular system.

\section{3-sectored case}

According to the spatial distribution of the users over the system, the mean squared path gain for the $K$ users in each cell belonging in the $l^{\text {th }}$ tier of interference from the receiver of interest is given by equations (13)-(15). We assume uniform user spatial distribution and the Rician fading environment with uniformly distributed phase. We also consider that there are always $\frac{6 \cdot l \cdot K}{3}=6 \cdot l \cdot \ddot{K}$ users in the $l^{t h}$ tier of interference. Hence, $\mathbb{E}\left[\ddot{\Lambda}_{\mathbf{y}}\right]$ becomes :

$$
\begin{aligned}
& \mathbb{E}\left[\ddot{\boldsymbol{\Lambda}}_{\mathbf{y}-\text { uni }}\right]= \\
& =\operatorname{diag}\left[1+G_{D,(3)} \ddot{K} P \cdot\left(\overline{\varsigma_{0-\text { uni }}^{2}}+\sum_{l=1}^{L} 6 \cdot l \cdot \overline{\varsigma_{l-\text { uni }}^{2}}\right)\right]
\end{aligned}
$$

Consequently, the asymptotic expression of the per-cell capacity for any finite number of users $K$ is given by:

$$
\begin{aligned}
\ddot{C}_{\text {uni }}=\lim _{N \rightarrow \infty} \frac{1}{N} \log \operatorname{det}\left(\mathbb{E}\left[\ddot{\mathbf{\Lambda}}_{\mathbf{y}-\text { uni }}\right]\right)= \\
=3 \cdot \log \left[1+G_{D,(3)} \ddot{K} P \cdot\left(\overline{\varsigma_{0-\text { uni }}^{2}}+\sum_{l=1}^{L} 6 \cdot l \cdot \overline{\varsigma_{l-\text { uni }}^{2}}\right)\right]
\end{aligned}
$$

as $\mathbb{E}\left[\ddot{\boldsymbol{\Lambda}}_{\mathbf{y}-\text { uni }}\right]$ has $3 N$ exactly same eigenvalues. One can easily reach to similar expressions for the per-cell capacity of the other two types of user spatial distribution.

$S$-sectored case
As the number of sectors, $S$, tends to infinity, we consider the fact that there will be $\frac{6 \cdot l \cdot K}{S}$ users in the $l^{\text {th }}$ tier of interference and that the expectation of covariance matrix of the output vector will have $S N$ exactly same eigenvalues. It can be shown that for uniformly distributed users the asymptotic capacity converges to:

$$
\begin{gathered}
\ddot{C}_{u n i}^{\star} \triangleq \lim _{S \rightarrow \infty} \ddot{C}_{\text {uni }}=\lim _{S \rightarrow \infty} S . \\
\cdot \log \left(1+\frac{G_{D,(S)} K P}{S}\left(\overline{\varsigma_{0-\text { uni }}^{2}}+\sum_{l=1}^{L} 6 \overline{\varsigma_{l-\text { uni }}^{2}}\right)\right)
\end{gathered}
$$

By solving the above equation we can see that the information theoretic capacity becomes a function of the directivity gain of the receiving antennas at the BSs given by:

$\ddot{C}_{u n i}^{\star}=\frac{G_{D,(S)} K P\left(\overline{\varsigma_{0-u n i}^{2}}+\sum_{l=1}^{L} 6 l \overline{\varsigma_{l-u n i}^{2}}\right)}{\ln (2)}$ bits/sec/Hz.

We can safely assume that, due to hardware limitations, the directivity gain does not grow linearly with the number of sectors. Thus, the above result indicates that even for infinite number of sectors, the system capacity tends always to a finite limit.

\section{MIMO Cellular Model}

The analysis above can be readily extended for the case where the BS receive antennas are ominidirectional and uncorrelated. We consider $M$ antennas at each BS and assuming uniform user spatial distribution within the Rician fading environment with uniformly distributed phases. The expectation of the covariance matrix of the output vector converges to an $M N \times M N$ diagonal matrix with $M N$ exactly same eigenvalues. Hence, the asymptotic expression of the per-cell capacity for a large number of users $K$ is given by:

$$
\begin{aligned}
& \hat{C}_{\text {uni }}=\lim _{N \rightarrow \infty} \frac{1}{N} \log \operatorname{det}\left(\mathbb{E}\left[\hat{\boldsymbol{\Lambda}}_{\mathbf{y}-\text { uni }}\right]\right)= \\
& =M \cdot \log \left[1+K P \cdot\left(\overline{\varsigma_{0-\text { uni }}^{2}}+\sum_{l=1}^{L} 6 \cdot l \cdot \overline{\varsigma_{l-\text { uni }}^{2}}\right)\right]
\end{aligned}
$$

In that case it can be observed that for infinite number of antennas at each BS $(M \rightarrow \infty)$ the capacity increases unbounded.

\section{REsults}

An important issue is to establish the relation of the various system modelling parameters with real-world scenarios so as to interpret the information theoretic results for these systems. To model the propagation in real-world systems more accurately we need to obtain a one-to-one correspondence between the simplified path loss model and the existing empirical models. As an example, we have selected two well-known empirical models for micro-cellular (Wideband PCS Microcell Model [13]) and macro-cellular (PCS extension to Hata model by COST-231 [13]) systems. Based on the limitations of the two models (in terms of the parameter ranges for which these models hold) we use the following parameters to approximate 


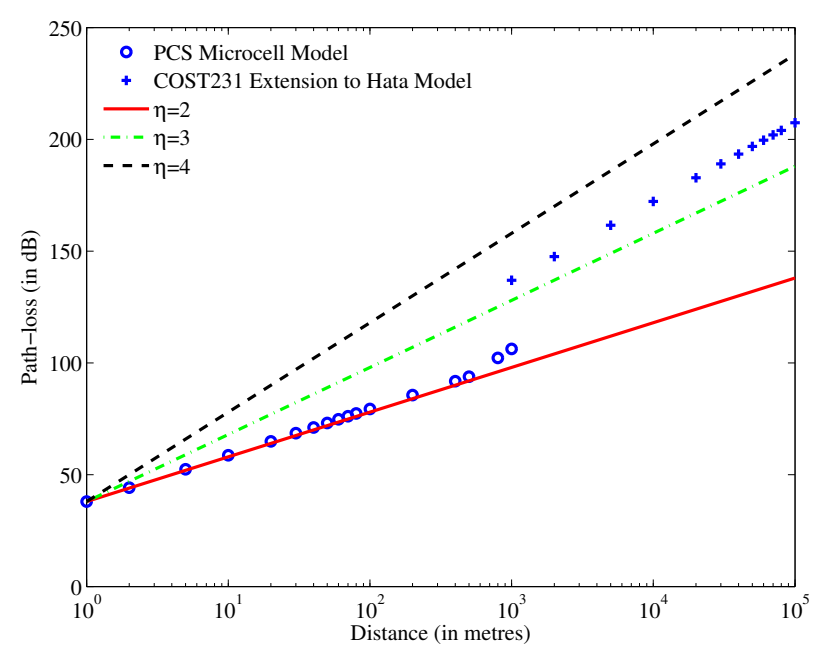

Fig. 6. Comparison of Empirical Models with Simplified Path Loss model

the path loss. For both models we use $f_{c}=1.9 \mathrm{GHz}, h_{r e}=$ $1.5 \mathrm{~m}$ and $L_{0}=38 \mathrm{~dB}$ where $f_{c}$ is the carrier frequency, $h_{r e}$ is the effective height of the receive antennas and $L_{0}$ is equal to the path loss in decibels at reference distance $D_{0}=1 \mathrm{~m}$. We use the minimum allowed transmit antenna height for the macrocellular $(30 \mathrm{~m})$ and the maximum allowed for the microcellular $(13.3 \mathrm{~m})$ system models. We assume a line-ofsight dual slope environment for microcellular system and a small/medium sized city environment for the macrocellular system. In order to see the relation of the simplified path loss model and the empirical models we plot the results obtained by the two empirical models and the results for simplified model with varying $\eta$ in Fig. 6 . It can be observed that the microcellular model suggests a smaller value of $\eta=2$ and the macrocellular model suggests a much larger value of $\eta=3.5$. We find the empirical value for the constant $L_{0}(-38 \mathrm{dBW})$ that achieves a close-fit between the simplified path loss model and the empirical models over a large range of distances.

In the following discussion, some interesting results on the information theoretic capacity of the sectorized planar cellular systems are presented. All the theoretical results have been verified by running Monte Carlo simulations to generate random fading coefficients user spatial distribution snapshots. The simulation capacity is obtained by finding the average over a large number of fading and user spatial distribution snapshots using the following equation:

$$
C_{\text {sim }}=\frac{1}{N} \mathbb{E}\left[\log _{2} \operatorname{det}\left(\boldsymbol{\Lambda}_{\mathbf{y}, \operatorname{sim}}\right)\right]
$$

where $\Lambda_{\mathbf{y} \text {,sim }}$ is the covariance matrix of appropriate size. For fair comparison the simulation results do not include shadowing. Nevertheless, as discussed in the previous section, valid insights for a shadow fading scenario can still be provided from the simplified path loss model. In Figures 7 and 9, alongside the uniform spatial distribution, the extreme cases of the cell-center and cell-edge spatial distributions are presented. For the truncated cell-centre spatial distribution, $\rho$ was considered to be equal to zero (all the users at the center of the cells), while for the truncated cell-edge spatial distribution,

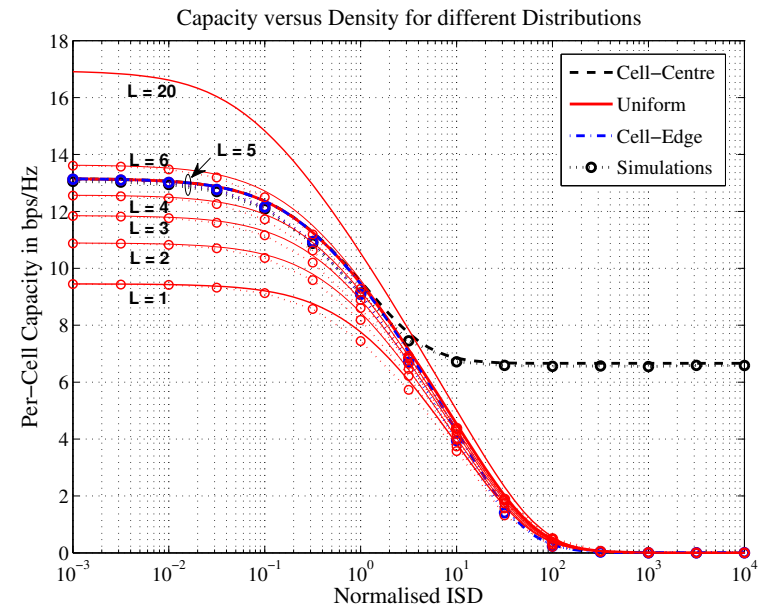

Fig. 7. Capacity per cell versus the normalised Inter Site Distance for the three different spatial distributions. The effect of different number of tiers of interference $(L=1-6,20)$ for uniform spatial distribution is illustrated. Normalised transmit power $=20 \mathrm{~dB}$ per cell, $\eta=2$.

$\rho$ was considered to be equal to $d_{0}$ (all the users at the edge of their cells).

In Fig. 7 the per-cell capacity of the generalised system model is plotted against the normalised Inter Site Distance (equal to the minimal diameter $2 r$ of the hexagonal cells) where the normalisation comes from the reference distance $D_{0}$ in equation (3). The small difference between the simulations and the analytical results can be attributed to the fact that for finding the simulation capacity $C_{\text {sim }}$, a finite system of $\left[\left(\sum_{l=1}^{L} 6 \cdot l\right)+1\right]$ cells with 100 users per cell was considered for each case of $L$, instead of the wrap-around toric model used for analysis. For $L>6$ the simulations become computational intensive, as the system becomes too large to be simulated, unlike the analytical method which can provide results even for very large $L$. In any case, as the system grows in size the edge effects should become even more negligible. The figure illustrates the behaviour of the capacity while the number of interfering tiers of cells changes for uniformly distributed users. It can be seen that for high values of normalised ISD the number of interfering tiers has no significant role on the capacity. On the contrary, as the system becomes more dense, the capacity increases with the number of the interfering tiers considered, suggesting that in a dense cellular system we can no longer use the model where the interference is considered to come only from the adjacent cells. In the same figure, the effect of user spatial distribution over the cells is illustrated. For $L=5$, it can be seen that for lower normalised ISD, the capacity for all spatial distributions coincide and reach the maximum possible value. On the other hand, as the size of the cells grows larger, the capacity decreases and the different spatial distributions correspond to different system capacity. Specifically, for uniform and celledge user spatial distributions, capacity tends to zero. For cellcentre spatial distribution, capacity decreases with the size of the cell and reaches to a specific non-zero value. This is due to the fact that the users of the cell of interest will always be close to their BSs no matter how large the size of the cell 

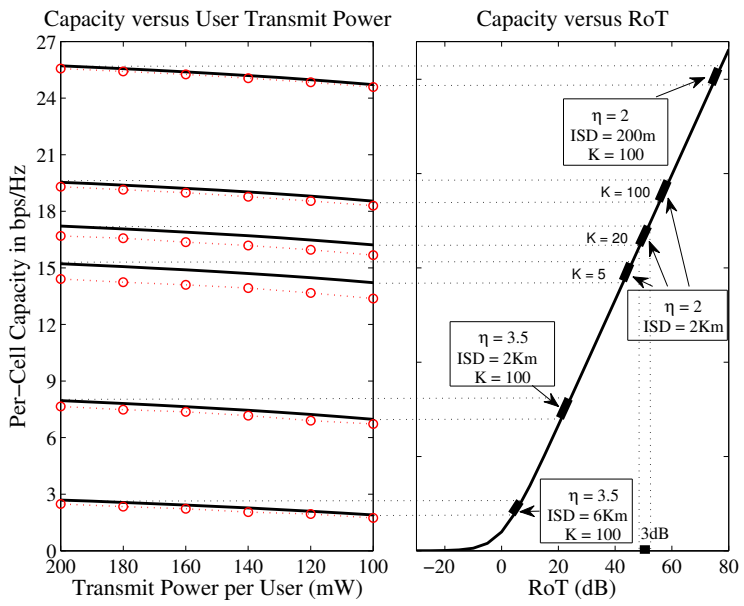

Fig. 8. Capacity per cell versus per user Transmit Power and versus RoT for different path loss exponents $(\eta=2,3.5)$ and different Inter Site Distances $(200 \mathrm{~m}, 2 \mathrm{Km}, 6 \mathrm{Km})$. Uniformly distributed users, $L=5, K=5,20,100$ users per cell with transmit power varying between $100-200 \mathrm{~mW}$.

will be. Note that, in all cases, uniform and cell-edge spatial distributions provide capacity very close to each other.

We consider a realistic scenario where circular cells have radii of $100 \mathrm{~m}-3 \mathrm{~km}$, the path loss at a reference distance of $1 \mathrm{~m}$ is $-38 \mathrm{~dB}$ (for a carrier frequency of $1.9 \mathrm{GHz}$ ) and the path loss exponent is either 2 or 3.5. The system has 5-100 UTs uniformly distributed per cell with transmit power constraint of $100-200 \mathrm{~mW}$ and thermal noise density of $-169 \mathrm{dBm} / \mathrm{Hz}$ with channel bandwidth of $5 \mathrm{MHz}$. Random received phases are assumed to plot the capacity in Fig. 8 against the Rise over Thermal (RoT, see Appendix B). By plotting the capacity against the RoT, a unified view of the capacity behaviour is obtained. Different parameters specify the operating range of RoT and hence the achieved capacity-range of the system. Capacity follows a Shannon-like function of the RoT:

$$
C=\log (1+\operatorname{RoT})
$$

Fig. 8 illustrates how the capacity-range for the system changes depending on the cell size, the path loss exponent, the number of users per cell and the per-user transmitted power. A maximum of 5 tiers of interfering cells is assumed for every cell. We note that the capacity increases for a relatively low path loss exponent and for small cell size. Furthermore, it is shown that increasing the number of users per cell increases the capacity (assuming joint decoding of the signals). We can also observe that a $3 \mathrm{~dB}$ increase in the transmit power (doubled from 100 to $200 \mathrm{~mW}$ ) does not have a significant effect on the capacity. Note that for larger number of users per cell (e.g. $K=100$ ) the simulation results reach closer to the analytical tight upper bound.

Fig. 9 compares the capacity obtained by the generalised, the sectorized and the MIMO system models. The results are obtained using the same real-world scenario described above. Results are obtained in the sectorized case for both worst and best case of antennas directivity gain (e.g. $G_{D,(3)}=1$ and $G_{D,(3)}=3$ respectively). The significant improvement (of the order of number of sectors) in the sectorized case,

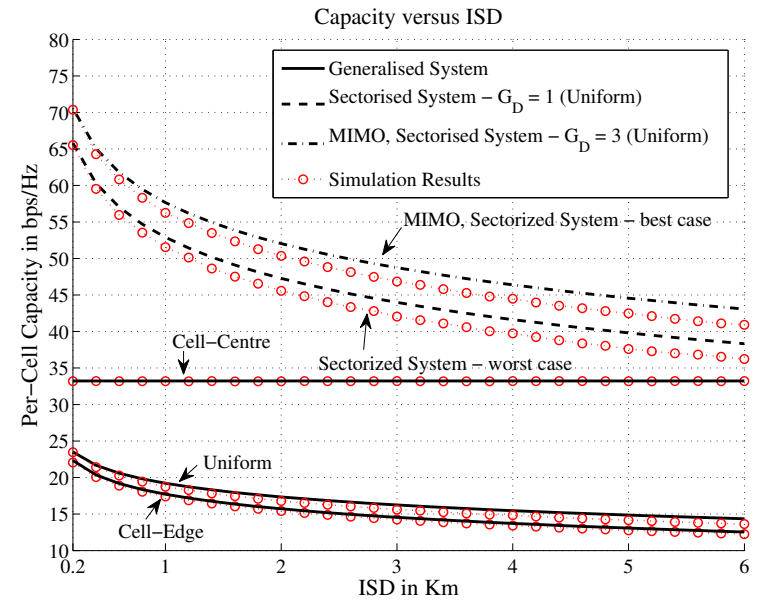

Fig. 9. Capacity per cell versus the Inter Site Distance for the Generalised, the Sectorized and the MIMO system model. $L=5, \eta=2, K=20$ users per cell with transmit power of $200 \mathrm{~mW}$ for every user.

even for $G_{D,(3)}=1$, can be attributed to increased degrees of freedom due to larger number of receiver antennas in the cellular system. Use of omnidirectional antennas provides a higher capacity that is attributed to the power gain obtained when the antennas receive the signals from all directions. Note that, for $G_{D,(3)}=3$, the sectorized system can provide the same capacity as the MIMO one. In the same figure, for the generalised system model, the capacity for different user spatial distributions is illustrated to emphasise on the fact that the user spatial distribution has an important role on the realworld system capacity.

\section{CONCLUSION}

We investigate the capacity of the planar cellular uplink. An average path loss approximation model was presented for the analysis of a 2D system where every BS receives signals from the same cell and the surrounding cells (arranged in multiple tiers of interference around the cell of interest). The size of the cells and hence the cell density is modelled as a variable. Assuming a joint decoder at the BSs ("hyper receiver" scheme) a tight upper bound, for the maximum per cell sumrate capacity, is provided. We extend the generalised cellular system to compare various system scenarios and their effect on capacity. System with single antenna at each BS yields minimum capacity. Degrees of freedom gain provides a higher capacity when a system with multiple receiving antennas is considered where each antenna is perfectly directional and the cell coverage is sectored. A further gain due to increased received power is obtained when the multiple BS antennas are considered omnidirectional and uncorrelated with each other or when the directivity gain of the directional antennas is considered larger than unity. It is also shown that increasing the number of sectors in each cell to a very high value, the capacity tends to a finite value which is formulated using asymptotic analysis of the system. When joint processing of all the receivers is considered for a cellular MAC, sectorization is not an optimum technique to obtain the highest capacity from the system when the directional antennas do not reach 
their maximum ideal performance. The reason is that the sectorization is essentially an interference avoidance technique which is not an optimum approach in the presence of joint decoding receiver. As a result, using a MIMO system with same infrastructure and no sectorization is preferred as this can provide higher system capacity when joint decoding is in operation. Nevertheless, the wide-spread deployment of sectorized cellular systems makes it an interesting question to quantify the capacity of such a system and in this paper we address this question. Furthermore, various parameters of a practical system that affect the per-cell capacity are identified and analysed. Specifically, these parameters are:

(1) the user spatial distribution over the cell,

(2) the cell size (and hence the cell density),

(3) the path loss exponent,

(4) the transmit power constraint of the users, and

(5) the number of users per cell.

These parameters have an effect on a unified parameter the Rise over Thermal (RoT) at each BS. This parameter is shown to directly control the information theoretic capacity of the system. Finally, it is argued that shadow fading can be captured in our empirical model and hence the simplified analytical path loss model can still provide valid insights for the shadow fading scenario. Nevertheless, the concept of the effect of shadow fading on the achievable capacity poses a very interesting question to be further analysed in our future approach.

\section{APPENDIX A \\ Output Covariance CONVERGENCE}

Consider the $N \times K N$ matrix $\mathbf{H}$ with Gaussian i.i.d. entries. Consider also the following multiplication:

$$
\boldsymbol{\Omega}=\mathbf{H H}^{\dagger}
$$

where $\mathbf{H}^{\dagger}$ is the $K N \times N$ Hermitian transpose matrix of $\mathbf{H}$. Each element of the matrix $\Omega$ is the result of the multiplication of a row of matrix $\mathbf{H}$ ( which is a $K N$ vector ) with a column of matrix $\mathbf{H}^{\dagger}$ ( again a $K N$ vector). Thus, each element of matrix $\Omega$ is the $K N$ sum of random variables multiplied with the conjugate transpose of other random variables:

$$
\omega_{i, j}=\sum_{k=1}^{K N}\left[h_{i, j, k} \cdot \hat{h}_{i, j, k}^{*}\right]
$$

where all of the random variables $h, h$ are assumed to follow the same distribution. The matrix $\Omega$ converges to a deterministic matrix equal to $\mathbb{E}[\Omega]$ if and only if all its elements converge, which means that the law of large numbers must apply to each element of $\boldsymbol{\Omega}$. For this, it is not sufficient that the dimensions of matrix $\mathbf{H}$ grow large. Instead, the horizontal dimension must grow much faster than the vertical dimension.

Hence, when $K \rightarrow \infty$ for every fixed $N$, the law of large numbers applies to each element of $\mathbf{H H}^{\dagger}$, i.e. $\omega_{i, j} \cong$ $K N^{2} \mathbb{E}\left[h \cdot h^{*}\right] \forall i, j$, and thus we can obtain a deterministic value for the expectation, $\mathbb{E}[\boldsymbol{\Omega}]$ and consequently for the capacity.

\section{APPENDiX B}

\section{DEFINITION OF ROT}

In the practical engineering design of cellular systems, the main figure of merit that determines the capacity (maximum reliable transmission rate with vanishingly small error rate) of a UT, is the SINR at the BS receiver, given as:

$$
\text { SINR }=\frac{\text { Total Wanted Received Power }}{\text { Total Unwanted Received Power }}=\frac{P_{R}}{I_{s}+\sigma_{0}^{2}}
$$

where $P_{R}$ is the wanted received power at the receiver of interest, $\sigma_{0}^{2}$ is the thermal AWGN power at the receiver and $I_{s}$ is the inter-cell and intra-cell interference received from other transmissions in the system. However, when joint decoding is considered for all the receivers in the system, the main figure of merit that determines the per-cell capacity is Rise over Thermal (RoT), defined as:

$$
\mathrm{RoT}=\frac{\text { Total Power Received }}{\text { Noise Power }}=\frac{P_{R}+I_{s}}{\sigma_{0}^{2}}
$$

which shows that the information theoretic approach of using a joint decoder has the potential of converting the conventionally "unwanted" interference into a "wanted" power. It shall be noted that the problem of finding the per-cell capacity of a cellular system can be greatly simplified by focusing on the single receiver node of the joint decoder and the associated RoT. Due to the symmetry of the problem (ignoring the edge effects) all receivers are identical and system capacity is simply the per-cell capacity times the number of cells. The mathematical formulation, analysis and simulation results presented in this paper back the heuristic idea described here.

\section{ACKNOWLEDGMENT}

The work reported in this paper has formed part of the "Fundamental Limits to Wireless Network Capacity" Elective Research Programme of the Virtual Centre of Excellence in Mobile \& Personal Communications, Mobile VCE, www.mobilevce.com. This research has been funded by the following Industrial Companies who are Members of Mobile VCE - BBC, BT, Huawei, Nokia, Nokia Siemens Networks, Nortel, Vodafone. Fully detailed technical reports on this research are available to staff from these Industrial Members of Mobile VCE. The authors would like to thank Prof. G. Caire and Prof. D. Tse for the fruitful discussions.

\section{REFERENCES}

[1] C. E. Shannon, "A mathematical theory of communication," Bell Systems Technical Journal, vol. 27, pp. 379-423, 1948.

[2] A. Wyner, "Shannon-theoretic approach to a Gaussian cellular multipleaccess channel," IEEE Transactions on Information Theory, vol. 40, no. 6, pp. 1713-1727, Nov. 1994.

[3] O. Somekh and S. Shamai, "Shannon-theoretic approach to a Gaussian cellular multiple-access channel with fading," IEEE Transactions on Information Theory, vol. 46, no. 4, pp. 1401-1425, July 2000.

[4] N. Letzepis and A. Grant, "Information capacity of multiple spot beam satellite channels," in Proc. 6th Australian Communications Theory Workshop, 2005, 2-4 Feb. 2005, pp. 168-174.

[5] K. S. Gilhousen, I. M. Jacobs, R. Padovani, A. J. Viterbi, L. A. Weaver, and C. Charles E. Whetlaey III, "On the capacity of a cellular CDMA system," IEEE Transactions on Vehicular Technology,, vol. 40, no. 2, pp. 303-312, 1991. 
[6] J. C. Liberti and T. S. Rappaport, "Analytical results for capacity improvements in CDMA," IEEE Transactions on Vehicular Technology, vol. 43, no. 3, pp. 680-690, 1994.

[7] S. V. Hanly and P. A. Whiting, "Information-theoretic capacity of multireceiver networks," Tellecommun. Syst., vol. Vol. 1, pp. pp. 1-42, 1993.

[8] D. Tse and P. Viswanath, Fundamentals of Wireless Communications. University Press, Cambridge, 2005.

[9] S. Jayaweera and H. Poor, "MIMO capacity results for Rician fading channels," in IEEE Global Telecommunications Conference, 2003. GLOBECOM '03, vol. 4, 1-5 Dec. 2003, pp. 1806-1810.

[10] A. Goldsmith, Wireless Communications. Cambridge University Press, 2005.

[11] T. S. Rappaport and L. B. Milstein, "Effects of path loss and fringe user distribution on CDMA cellular frequency reuse efficiency," IEEE Global Telecommunications Conference, 1990. GLOBECOM '90, vol. 1, pp. 500-506, 1990.

[12] I. E. Telatar, "Capacity of multi-antenna Gaussian channels," European Transactions on Telecommunications, vol. 10, no. 6, pp. 585-595, November 1999.

[13] T. S. Rappaport, Ed., Wireless Communications: Principles and Practice. Upper Saddle River, NJ, USA: Prentice-Hall, Inc., 1995.

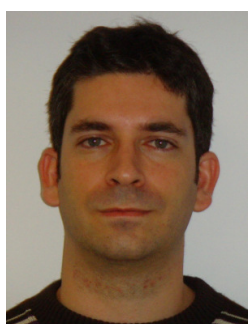

Efstathios Katranaras was raised in Levadia, Greece. He received the B.Sc. degree in electrical and computer engineering from the National Technical University of Athens (NTUA), Greece, in 2005 and the M.Sc. degree in mobile communications from the University of Surrey, U.K., in 2006. Currently, he is a Member of the Centre for Communications Systems Research, University of Surrey. His main research interests are in the area of wireless systems and focused on the evaluation of fundamental capacity limits.

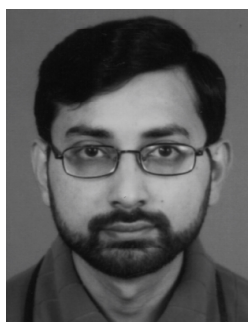

Muhammad Ali Imran received his B.Sc. degree in Electrical Engineering from University of Engineering and Technology Lahore, Pakistan , in 1999, and the M.Sc. and Ph.D. degrees from Imperial College London, UK, in 2002 and 2007, respectively. He is currently a research fellow at the Centre for Communication Systems Research (CCSR) at the University of Surrey, UK. His main research interests include the analysis and modelling of the physical layer, resource allocation in multiuser communication netlimits of wireless networks. works and evaluation of the fundamental capacity

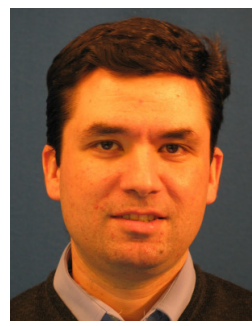

Costas Tzaras received the B.Sc. degree in physics from the University of Thessaloniki, Greece and the M.Sc. and Ph.D. degrees in Mobile and satellite communications from the University of Surrey, U.K. In 1997, he worked as a Research Fellow at the University of Surrey and later he was employed by Vodafone R\&D in the Radio Modelling Group to act as the Principal Investigator in radio modelling issues across the Vodafone Group. In 2002, he returned to the University of Surrey as a Lecturer in Mobile Communications and he continued to lead the Antennas and Propagation Team at Vodafone Global. Since 2007, he is working for Kimatica Ltd which is a software company that specialises in the field of radio modelling. His main interests lie in the field of wireless technology with emphasis on radio channel modelling, network planning and optimization and capacity investigations on wireless systems. 